\title{
Programa de Pós-Graduação em Enfermagem na Saúde do Adulto da Escola de Enfermagem da USP
}

POST-GRADUATION PROGRAM IN ADULT HEALTH NURSING FROM THE NURSING SCHOOL OF UNIVERSITY OF SAO PAULO

PROGRAMA DE POST-GRADO EN ENFERMERÍA EN LA SALUD DELADULTO DE LA ESCUELA DE ENFERMERÍA DE LA UNIVERSIDAD DE SÃO PAULO

\section{Cibele Andrucioli de Mattos Pimenta ${ }^{1}$, Diná de Almeida Lopes Monteiro da Cruz ${ }^{2}$, Katia Grillo Padilha ${ }^{3}$}

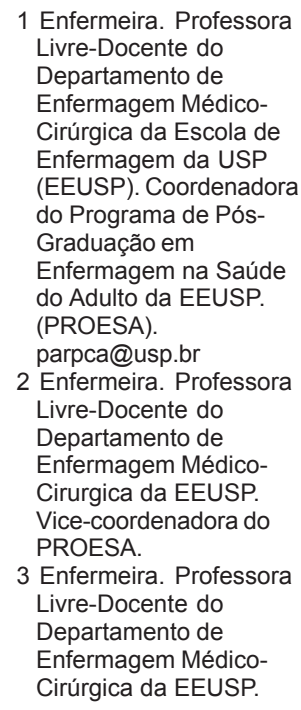

3 Enfermeira. Professora Livre-Docente do Departamento de Enfermagem MédicoCirúrgica da EEUSP.

\begin{abstract}
RESUMO
O Programa de Pós-Graduação em Enfermagem na Saúde do Adulto da Escola de Enfermagem da USP (PROESA) nasceu da fusão das Áreas de Concentração "Fundamentos de Enfermagem" e "Enfermagem na Saúde do Adulto Institucionalizado", anteriormente pertencentes ao Programa de Pós-Graduação da referida Escola. Há carência de descrição de programas de pósgraduação em enfermagem no Brasil, o que dificulta a organização de novas propostas e o aperfeiçoamento dos existentes. O objetivo deste artigo é apresentar a missão, o foco, as linhas de pesquisa, os eixos norteadores e o conjunto de disciplinas do PROESA.
\end{abstract}

\section{DESCRITORES}

Educação de pós-graduação em enfermagem.

Desenvolvimento de programas. Educação superior.

Saúde do adulto.

\begin{abstract}
The Post-graduation Program in Adult Health Nursing from the Nursing School of University of Sao Paulo resulted from the fusion of two concentration areas: "Fundamental Nursing" and "Adult Health Nursing", which were part of the Postgraduation Program of the aforementioned School. Descriptions of Post-graduation Programs could facilitate the organization of new program proposals and the improvement of the current ones, but there are a few descriptions in Brazil. This article presents the mission, the focus, the guiding axes, the research lines, and the disciplines of the Adult Health Nursing Post-graduation Program of the Nursing School of University of Sao Paulo.
\end{abstract}

\section{DESCRIPTORS}

Nursing Post-graduation

Education.

Program development.

Higher education.

Adult health.

\section{RESUMEN}

El Programa de Post Grado en Enfermería en la Salud del Adulto de la Escuela de Enfermería de la Universidad de São Paulo (PROESA) nació de la unión de las áreas de concentración "Fundamentos de Enfermería" y "Enfermería en la Salud del Adulto Institucionalizado", anteriormente pertenecientes al Programa de Post Grado en Enfermería de la referida Escuela. En el Brasil hay carencia de descripción de los Programas de Post Grado en Enfermería hecho que dificulta la organización de nuevos Programas y perfeccionamiento de los existentes. Este artículo presenta la misión, el foco, las líneas de investigación, los ejes directrices y el conjunto de disciplinas del PROESA.

\section{DESCRIPTORES}

Educación de post grado en enfermería.

Desarrollo de programas.

Educación superior. Salud del adulto. 


\section{INTRODUÇÃO}

A Escola de Enfermagem da Universidade de São Paulo (EEUSP) foi criada em 1942. É uma instituição de ensino superior com tradição na formação de bacharéis, especialistas, mestres e doutores na área de enfermagem, em âmbito nacional e na América do Sul. Possui infra-estrutura humana e material adequadas para o desenvolvimento das atividades de ensino e pesquisa no âmbito da pós-graduação lato sensu e stricto sensu.

A pós-graduação stricto sensu da EEUSP, nível mestrado, iniciou suas atividades em 1973 e, em 1982, a Escola, em parceria com a Escola de Enfermagem de Ribeirão Preto da USP, iniciou o Programa Interunidades de Doutorado em Enfermagem. Em 1999 o curso de mestrado era constituído por sete áreas de concentração: Administração de Serviço de Enfermagem, Enfermagem Psiquiátrica, Enfermagem Pediátrica, Enfermagem Obstétrica, Enfermagem em Saúde Coletiva, Fundamentos de Enfermagem e Enfermagem na Saúde do Adulto Institucionalizado. Em 1987 iniciou-se o curso de doutorado pela EEUSP.

A área de Fundamentos de Enfermagem, criada em 1973, foi a pioneira no estabelecimento do Programa de Pós-Graduação da EEUSP, e tinha como foco de estudo os modelos conceituais de enfermagem e a operacionalização destes na clínica. A área de Enfermagem na Assistência ao Adulto Institucionalizado, criada em 1995, tinha como foco de estudo o cuidado ao adulto em instituições de saúde. As duas áreas pertenciam ao Departamento de Enfermagem MédicoCirúrgica (ENC) e eram desenvolvidas pelo mesmo grupo de docentes.

A partir de 1995 o número de doutores no Departamento ENC aumentou consideravelmente com significativa ampliação do número de disciplinas na pós-graduação, maior especificidade e produção científica em torno de linhas de pesquisa que evidenciavam a vocação deste grupo. Significativa parcela das disciplinas e dos temas de pesquisa, que foram se configurando com os novos doutores, utilizavam referenciais teóricos e intervenções que tinham como substrato o estudo de temas avançados sobre o cuidado ao adulto, desenvolvidos de modo complementar nas duas áreas. Percebia-se a confluência das duas áreas e a distinção destas das demais que compunham o Programa de Pós-graduação em Enfermagem da EEUSP. O processo natural de diferenciação criou a necessidade de se reorganizar a estrutura da pós-graduação stricto sensu da Escola, para acolher e fortalecer as novas singularidades e permitir a melhora do conjunto.

A Pós-Graduação da Escola discutiu, em fóruns, o aperfeiçoamento acadêmico e administrativo do programa de pós-graduação. Dessas discussões, reconheceu-se a convergência das duas áreas (Fundamentos de Enfermagem e Enfermagem na Saúde do Adulto Institucionalizado), a melhor conformação e especificidade que adviria da união delas, a existência de corpo docente com produção científica significativa e a hipertrofia da estrutura de Pós-Graduação da Escola. Diante de tais fatos, decidiu-se que a fusão das áreas Fundamentos de Enfermagem e Enfermagem na Assistência ao Adulto Instituciona-lizado e a criação de um novo programa traria benefícios de natureza científica, acadêmica e administrativa para a Pós-Graduação. Tais benefícios, maior identidade e verticalização do conhecimento, composição de linhas de pesquisa mais específicas e agilidade administrativa, estender-se-iam ao programa já existente e ao novo programa.

Após estudos que demonstraram que a proposição de um novo programa traria benefícios ao programa já existente, foi organizada a proposta do Programa de Pós-Graduação em Enfermagem na Saúde do Adulto da EEUSP. O Programa de Pós-Graduação em Enfermagem na Saúde do Adulto da EEUSP (PROESA) foi aprovado em todas as instâncias da EEUSP e da Universidade de São Paulo. Em julho de 2002 foi recomendado pelo Conselho Técnico Científico (CTC) da CAPES com os cursos de Mestrado e Doutorado (ofício CTC/CAPES no $163 / 2002$ ).

O objetivo deste artigo é apresentar a proposta do PROESA. Considerando-se a quase inexistência de artigos que apresentem propostas de cursos de pós-graduação stricto sensu, julga-se que o relato de tal experiência possa ser útil para a comunidade interessada na reflexão e organização de programas de pós-graduação em enfermagem.
Programa de PósGraduação em Enfermagem na Sáude do Adulto da Escola de Enfermagem da USP 
Cibele A. de Mattos Pimenta Diná de A.L.M. da Cruz Kátia Grillo Padilha

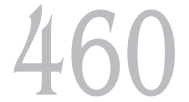

Rev Esc Enferm USP 2004; 38(4): 458-66.

\section{JUSTIFICATIVA E INSERÇÃO REGIONAL DO PROESA}

Diversos fatores motivaram e dão sustentação ao Programa de Pós-Graduação em Enfermagem na Saúde do Adulto.

Há necessidade de construção do conhecimento sobre o cuidado avançado de enfermagem em situações clínicas específicas e há carência desta abordagem nos programas de pós-graduação existentes, em âmbito regional e nacional.

Análises de extenso volume de informações permitem observar que existe coerência entre a produção intelectual do corpo docente, as linhas de pesquisa e a estrutura curricular do presente Programa, onde temas avançados sobre o cuidado ao adulto são o substrato para o desenvolvimento de cientistas e professores, e há significativo número de doutores, atuando em regime de dedicação integral ao ensino e à pesquisa, com expressiva produção científica, experiência comprovada na formação de mestres e doutores e participação em grupos de pesquisa.

As escolas de ensino superior necessitam de corpo docente capacitado para o ensino e a pesquisa de questões vinculadas às necessidades e características regionais para que sua atuação tenha o alcance social esperado para este nível de ensino. A procura de pós-graduação nas área de Enfermagem na Saúde do Adulto tem sido expressiva e a EEUSP conta com excelente infra-estrutura acadêmica e administrativa.

A região sudeste concentra cerca de $42 \%$ da população brasileira ${ }^{(1)}$ e $50 \%$ dos enfermeiros do Brasil( ${ }^{(2)}$. No Estado de São Paulo vive cerca de $22 \%$ da população brasileira ${ }^{(1)} \mathrm{e}$ $25 \%$ do total nacional de enfermeiros ${ }^{(2)}$.Dos cerca de 274 cursos de graduação em enfermagem do país no ano de $2003^{(3)}, 132$ (48,2\%) estão na região Sudeste e $69(25,2 \%)$ no Estado de São $\mathrm{Paulo}^{(3)}$. A região sudeste é centro formador de referência para todo o Brasil e países da América Latina.

Na região sudeste, os problemas de saúde são de natureza múltipla e paradoxal. $\mathrm{O}$ perfil populacional e de morbi-mortalidade assemelha-se ao de regiões desenvolvidas, coexistindo aspectos característicos de regiões em desenvolvimento. Há grande número de adultos com doenças crônicas, com mani- festações agudas, vítimas de trauma e em envelhecimento. As práticas do sistema de saúde regional demandam profissionais de enfermagem altamente qualificados para pesquisar e ensinar sobre o cuidado avançado do adulto com afecções crônicas e agudas específicas.

Tais fatos indicam que o Programa em questão preenche uma lacuna existente e apresenta condições de sustentar os processos de geração de conhecimento e de formação de pessoal na área do cuidado avançado em enfermagem na saúde do adulto. Cuidado avançado em enfermagem, segundo a definição operacional adotada para o Programa, é aquele que expressa inovações teóricas, tecnológicas e operacionais na concepção dos fenômenos, intervenções e resultados em enfermagem.

\section{MISSÃO, NÚCLEO CENTRAL, LINHAS DE PESQUISA, EIXOS TEMÁTICOS E CONJUNTOS DE DISCIPLINAS}

O PROESA tem como missão capacitar enfermeiros para os processos de produção de conhecimento, processos de produção de ensino superior e processos de atualização científica e de aperfeiçoamento em tecnologias do cuidar, no âmbito da Enfermagem na Saúde do Adulto, integrando conhecimentos da ciência de enfermagem aos de outras disciplinas. Tal missão tem como base o proposto por Botomé( ${ }^{(4)}$, ao abordar um currículo estratégico para o desenvolvimento de Programas de Pós-Graduação em qualquer área do conhecimento.

O conhecimento produzido deve ser inovador e rigorosamente construído, demonstrando a habilidade do aluno em analisar a literatura em pesquisa, sintetizar achados e desenhar estudos utilizando metodologia adequada e respeitando os aspectos éticos envolvidos. Os educadores devem demonstrar habilidades de reflexão, estar atentos às necessidades do ensino superior e ser capazes de propor e avaliar planos de ensino que atendam a essas necessidades. Os egressos do programa devem ter desenvolvido comportamentos de contínua atualização científica e constante aperfeiçoamento em tecnologias do cuidar que os tornem capazes de impulsionar a pesquisa, a educação e o cuidado de enfermagem em práticas clínicas 
avançadas, dentro do escopo legal e ético da profissão. Tais características constituem o perfil dos egressos.

Cuidar do Adulto em Enfermagem é um processo que articula fenômenos, intervenções e resultados no âmbito da ciência de enfermagem, pertinentes à prevenção, tratamento e reabilitação dos agravos à saúde, tendo como finalidade a promoção à saúde do adulto ${ }^{(5-7)}$.

O núcleo central de estudo do Programa é o Cuidar do Adulto em Enfermagem. Cuidar do Adulto em Enfermagem é um processo que se desenvolve junto ao indivíduo - paciente, cliente e família - que articula a identificação de fenômenos, implementação de intervenções e avaliação de resultados, no âmbito da ciência da enfermagem, pertinentes à prevenção, tratamento e reabilitação nos agravos à saúde. Alicerça-se no modelo clínico de cuidar e compreende funções de cuidado direto e planejamento da assistência à saúde, por meio de ações independentes e em colaboração com a equipe multiprofissional, em âmbito institucional e domiciliar.

O adulto, alvo deste cuidar, é o indivíduo que alcançou a maturidade biológica e psicossocial, que na nossa sociedade é aceita como ocorrendo em torno dos 18-21 anos, com agravos ou risco de agravos à saúde, agudos ou crônicos, cuja gênese e expressão incluem elementos biológicos, comportamentais, emocionais e socioculturais. Excluem-se os adultos cuja demanda de cuidado foca-se, primordialmente, no ciclo gravídico-puerperal, nos distúrbios psiquiátricos e nas doenças transmissíveis.

Os fenômenos pertinentes ao conhecimento do cuidar em Enfermagem na Saúde do Adulto compreendem situações clínicas em que há contribuição da enfermagem para o alcance das melhores condições de saúde. Esses fenômenos estendem-se às dimensões fisiológicas, comportamentais, emocionais e socioculturais, envolvidas no processo saúde-doença do ser humano. A produção de conhecimento sobre os fenômenos de enfermagem refere-se ao estudo sistemático de formulação, mensuração e validação de conceitos, teorias e classificações.

As intervenções pertinentes ao cuidar em Enfermagem na Saúde do Adulto compreendem conjuntos de ações que visam modular os fenômenos de modo a se alcançar as melhores condições de saúde. Essas intervenções são ações diretas, independentes e complementares, realizadas junto ao adulto e ao meio em que ele se insere, e se estendem às dimensões fisiológicas, comportamentais, emocionais e socioculturais. A produção de conhecimento sobre intervenções de enfermagem envolve estudos clínicos que produzam evidências científicas sobre os efeitos das intervenções nos fenômenos de interesse.

Os resultados pertinentes ao cuidar em Enfermagem na Saúde do Adulto compreendem a definição das modificações que se quer alcançar no fenômeno com as intervenções propostas e a validação quanto ao ajuste do binômio fenômeno-intervenção. A produção de conhecimento sobre resultados envolve estudos sobre mensuração, classificação, efetividade e eficácia das intervenções estudadas.

No Programa de Pós-Graduação em Enfermagem na Saúde do Adulto articulam-se três eixos temáticos (dimensões): a dimensão do cuidar clínico, a do contexto onde esse cuidado se efetiva e a dimensão da produção de ensino superior. Esses eixos organizam três conjuntos de disciplinas e cinco linhas de pesquisa.

A definição das cinco linhas de pesquisa, Cuidar do Adulto com Afecções Crônicas não Transmissíveis, Cuidar do Adulto com Afecções Agudas e Críticas, Tecnologia na Saúde do Adulto, Processo Ensino-Aprendizagem na Saúde do Adulto, Dinâmicas de Trabalho na Saúde do Adulto está a seguir apresentada.

Cuidar do adulto com afecções crônicas não transmissíveis compreende estudos sobre fenômenos, intervenções e resultados pertinentes ao cuidar do adulto com afecções crônicas não transmissíveis, na perspectiva da promoção da saúde (prevenção, tratamento e reabilitação). Cuidar do adulto com afecções agudas e críticas compreende estudos sobre fenômenos, intervenções e resultados pertinentes ao cuidar do adulto com afecções agudas e críticas, na perspectiva da promoção da saúde (prevenção e tratamento). Tecnologia na saúde do adulto compreende estudos sobre a criação, desenvolvimento, adaptação e avaliação de modelos, instrumentos e procedimentos assistenciais relaciona-
Programa de PósGraduação em Enfermagem na Sáude do Adulto da Escola de Enfermagem da USP 
Cibele A. de Mattos Pimenta Diná de A.L.M. da Cruz Kátia Grillo Padilha

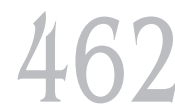

Rev Esc Enferm USP 2004; 38(4): 458-66. dos ao cuidar da saúde do adulto. Processo ensino-aprendizagem na saúde do adulto compreende estudos sobre conceitos, modelos, processos e métodos envolvidos na capacitação cognitiva, afetiva e comportamental do aluno e do profissional para cuidar do adulto e Dinâmicas de trabalho na saúde do adulto compreende estudos sobre conceitos, modelos, processos e métodos envolvidos nas relações de trabalho entre profissionais, clientes e instituições no contexto da saúde do adulto.

A articulação entre eixos temáticos, disciplinas e linhas de pesquisa encontra-se a seguir explanada.

No eixo cuidar clínico incluem-se três linhas de pesquisa: Cuidar do Adulto com Afecções Crônicas não Transmissíveis, Cuidar do Adulto com Afecções Agudas e Críticas e Tecnologia na Saúde do Adulto. Tais linhas coadunam-se com o perfil de morbimortalidade do adulto, em que prevalecem as doenças crônicas não transmissíveis e as causas externas. As situações de cuidar de indivíduos com agravos agudos e crônicos, por apresentarem especificidade na gênese e manifestação dos fenômenos, bem como nas intervenções requeridas, demandam conhecimentos diferenciados de modelos, instrumentos e procedimentos assistenciais, que compreendem tecnologias materiais e não materiais.

O cuidar do adulto em enfermagem é desenvolvido em diferentes contextos assistenciais (ambulatórios, unidades de emergência, de internação, domicílio), por um grupo de pessoas (equipe de enfermagem), imbricado no fazer de outros profissionais (equipe de saúde) e interrelacionado à estrutura política, filosófica e econômica das instituições de saúde e dos órgãos governamentais. Essa complexa rede de relações, com referenciais teóricos advindos principalmente da socio$\operatorname{logia}{ }^{(8)}$ e psicologia social ${ }^{(9-11)}$, que constitui a dimensão do contexto do cuidar, influi na natureza e manifestação dos fenômenos, na conceptualização e operacionalização das intervenções e no estabelecimento e mensuração dos resultados. Disso decorre a compreensão de que o processo de cuidar do adulto inclui aspectos relativos às relações entre os profissionais, destes com os clientes e com a instituição. A linha de pesquisa Dinâmicas de Trabalho na Saúde de Adulto refere-se a estudos sobre esses aspectos.
A terceira dimensão é a produção de ensino superior em Enfermagem na Saúde do Adulto. Visa articular a transformação de conhecimentos em comportamentos, e de comportamentos profissionais em novos conhecimentos, a partir das diversas linhas de pensamento da educação ${ }^{(12-14)}$. A linha de pesquisa Processo Ensino-Aprendizagem na Saúde do Adulto refere-se a estudos sobre a capacitação cognitiva, afetiva e comportamental do aluno e do profissional para o cuidar do adulto.

O PROESA conta com 32 disciplinas que compõem três conjuntos articulados aos eixos temáticos cuidar clínico, contexto do cuidar e produção de ensino superior, que foram nominados: processos de produção de conhecimento, modelos teóricos sobre o cuidar em condições clínicas específicas e ensino superior. A relação nominal das disciplinas pode ser observada no Quadro 1 (Anexo).

Observando-se o Quadro 1 nota-se que as disciplinas relativas à produção de conhecimento articulam-se com os 3 eixos temáticos. O conjunto de disciplinas relativas aos Modelos teóricos sobre o cuidar em condições clínicas específicas articulam-se aos eixos cuidar clínico e contexto do cuidar, e constituem a maior parte das disciplinas (26/32), o que demonstra a vocação deste Programa.

As linhas de pesquisa Cuidar do Adulto com Afecções Crônicas, Cuidar do Adulto com Afecções Agudas e Críticas e Tecnologia na Saúde do Adulto, articulam-se aos eixos Cuidar Clínico. As linhas de pesquisa Processos de Trabalho na Saúde do Adulto e Tecnologia na Saúde do Adulto, articulamse ao eixo temático Contexto do Cuidar. A linha Processos de Ensino-Aprendizagem do Adulto articula-se ao eixo processos de produção de ensino superior.

Há 26 disciplinas no conjunto Modelos Teóricos sobre o Cuidar em Condições Clínicas Específicas; destas, 20 estão vinculadas ao eixo temático Cuidar Clínico e 06 ao eixo temático Contexto do Cuidar. Há 05 disciplinas do conjunto Processos de Produção de Conhecimento que se vinculam aos eixos Cuidar Clínico, Contexto de Cuidar e Produção de Ensino Superior e há 1 disciplina sobre ensino superior, que se relaciona ao eixo temático produção de ensino superior. 
Além das 32 disciplinas específicas do PROESA, o aluno pode acessar disciplinas oferecidas em outros programas de pós-graduação, desta ou de outras unidades da Universidade de São Paulo, visando complementar sua formação.

As disciplinas relativas aos processos de produção de conhecimento visam capacitar o pós-graduando a delimitar problemas de pesquisa, a acessar as fontes de conhecimento, a identificar a maturidade e as lacunas do conhecimento na área, a analisar o método da ciência, a metodologia científica e os aspectos éticos dos estudos e a interpretar criticamente desenhos e resultados de pesquisa. Objetivam, também, capacitar o aluno para planejar desenhos de pesquisa e analisar as relações entre ciência, universidade e sociedade.

O segundo conjunto de disciplinas refere-se aos modelos teóricos sobre o cuidar em condições clínicas específicas. Tratam de conteúdos sobre os processos de atualização científica e de aperfeiçoamento em tecnologias do cuidar em enfermagem em situações clínicas específicas. Tal conjunto objetiva capacitar o pós-graduando a atualizar-se continuamente em relação ao conhecimento existente para lidar com o seu tema de investigação na área do cuidar em enfermagem do adulto. Essas disciplinas servem de substrato para a aprendizagem dos processos de produzir conhecimento e dos processos de ensinar, além de permitirem o domínio sobre a tecnologia e os avanços do conhecimento na área do cuidar em enfermagem na saúde do adulto. Visam capacitar o pósgraduando para sistematizar o conhecimento e analisar os avanços científicos sobre o objeto de seu estudo, nos limites da área Enfermagem na Saúde do Adulto e também capacitar o pós-graduando para integrar o conhecimento existente na área de Enfermagem na Saúde do Adulto a diferentes áreas do saber, bem como gerenciar sistemas de pesquisa em relação ao fenômeno de estudo. Objetivam, ainda, capacitar o aluno a refletir sobre o impacto das pesquisas na área de conhecimento específico e em áreas correlatas e na sociedade.

A disciplina relativa ao ensino superior em enfermagem objetiva capacitar o pós-graduando a identificar as necessidades de aprendizagem, programar, implementar e ava- liar programas de ensino na área, fundamentados na produção científica sobre o cuidar em enfermagem e sobre o ensino superior. Visa, também, capacitar o aluno para refletir sobre os limites e as perspectivas do ensino superior em Enfermagem na Saúde do Adulto e para articular as pesquisas sobre ensino desenvolvidas na área de enfermagem às realizadas em outras áreas.

O Programa de Pós-Graduação em Enfermagem na Saúde do Adulto da EEUSP atende às normas do Regimento de Pós-Graduação da USP e da Comissão de Pós-Graduação EEUSP, no tocante ao número de créditos necessários, prazos, critérios de seleção de candidatos, de credenciamento de orientadores, entre outras. Tal Regimento pode ser encontrado no portal da USP (www.usp.br).

\section{OBJETIVOS DO PROESA}

\section{O PROGRAMA ENFERMAGEM NA} SAÚDE DO ADULTO, para atender à missão de capacitar enfermeiros para os processos de produção de conhecimento, processos de produção de ensino superior e processos de atualização científica e de aperfeiçoamento em tecnologias do cuidar, no âmbito da enfermagem na saúde do adulto, tem como objetivos que os alunos sejam capazes de:

- produzir conhecimento inovador e rigorosamente construído, respeitando os aspectos éticos envolvidos;

- demonstrar habilidades de reflexão e de intervenção educativa, atreladas às necessidades do ensino superior em enfermagem;

- desenvolver comportamentos de contínua atualização científica e constante aperfeiçoamento em tecnologias do cuidar.

Os objetivos dos cursos de Mestrado e Doutorado do Programa de Pós-Graduação em Enfermagem na Saúde do Adulto estão descritos na Tabela 1.

A discriminação dos objetivos para o mestrado e doutorado tem a finalidade de nortear o oferecimento de atividades pelo programa e o plano de trabalho dos alunos. Os alunos de mestrado poderão avançar mais ou menos nos objetivos estabelecidos para o doutorado.
Programa de PósGraduação em Enfermagem na Sáude do Adulto da Escola de Enfermagem da USP 
Cibele A. de Mattos Pimenta Diná de A.L.M. da Cruz Kátia Grillo Padilha

Tabela 1 - Objetivos do curso de Mestrado e Doutorado do PROESA. (São Paulo 2002)

\begin{tabular}{|c|c|c|}
\hline OBJETIVOS & Mestrado & Doutorado \\
\hline Acessar as fontes do conhecimento & $\mathrm{X}$ & $\mathrm{X}$ \\
\hline $\begin{array}{l}\text { Sistematizar o conhecimento e analisar os avanços científicos } \\
\text { sobre o objeto de estudo }\end{array}$ & $\mathrm{X}$ & $\mathrm{X}$ \\
\hline Identificar a maturidade e as lacunas do conhecimento na área & $\mathrm{X}$ & $\mathrm{X}$ \\
\hline Delimitar problemas de pesquisa & $\mathrm{X}$ & $\mathrm{X}$ \\
\hline $\begin{array}{l}\text { Analisar o método da ciência, a metodologia científica e os } \\
\text { aspectos éticos dos estudos }\end{array}$ & $\mathrm{X}$ & $\mathrm{X}$ \\
\hline Interpretar criticamente desenhos e resultados de pesquisa & $\mathrm{X}$ & $\mathrm{X}$ \\
\hline Planejar desenhos de pesquisa & & $\mathrm{X}$ \\
\hline $\begin{array}{l}\text { Demonstrar ter adquirido perspectivas múltiplas do } \\
\text { conhecimento integrando o existente na área de } \\
\text { Enfermagem na Saúde do Adulto a diferentes áreas do saber }\end{array}$ & & $\mathrm{X}$ \\
\hline $\begin{array}{l}\text { Refletir sobre o impacto das pesquisas em áreas } \\
\text { correlatas e na sociedade }\end{array}$ & & $\mathrm{X}$ \\
\hline $\begin{array}{l}\text { Gerenciar sistemas de pesquisa em relação ao fenômeno } \\
\text { de estudo }\end{array}$ & $\mathrm{X}$ & $\mathrm{X}$ \\
\hline Analisar as relações entre ciência, universidade e sociedade & & $\mathrm{X}$ \\
\hline $\begin{array}{l}\text { Identificar as necessidades de aprendizagem, programar, } \\
\text { implementar e avaliar programas de ensino na área }\end{array}$ & & $\mathrm{X}$ \\
\hline $\begin{array}{l}\text { Articular as pesquisas sobre ensino desenvolvidas na } \\
\text { área de enfermagem às realizadas em outras áreas }\end{array}$ & & $\mathrm{X}$ \\
\hline
\end{tabular}

\section{CONSIDERAÇÕES FINAIS}

O desenvolvimento da Pós-Graduação em Enfermagem no Brasil é significativo. Nos últimos anos houve intenso esforço para a reorganização dos programas existentes e proposição de novos. No entanto, a descrição dessas experiências de revisão e proposição de novos programas é rara na literatura. Julga-se que apresentar a experiência do Departamento de Enfermagem Médico-Cirúrgica da EEUSP na organização do Programa de PósGraduação em Enfermagem na Saúde do Adulto (PROESA) pode contribuir com a discussão sobre pós-graduação em enfermagem no Brasil.

\section{REFERÊNCIAS}

(1) Fundação Instituto Brasileiro de Geografia e Estatística. Pesquisa Nacional por Amostra de Domicílios: PNAD 2000. [online]. Rio de Janeiro; 2001. Disponível em: $<$ http://www1. ibge.gov.br> (01 nov. 2001)

(2) Conselho Federal de Enfermagem. Dados estatísticos. [online]. São Paulo; 2004. Disponível em :<http://www.portalcofen.gov.br $>(10$ fev. 2004)

(3) Ministério da Educação. Educação superior. [online]. Brasília; 2004. Disponível em: $<$ http://www.mec.gov.br> (30 jan. 2004)
(4) Botomé SP. Um currículo estratégico para o desenvolvimento de mestrados e doutorados em diferentes áreas do conhecimento. Educ Bras 1999; 21(42):97-120.

(5) North American Nursing Diagnosis Association (NANDA). International. Nursing Diagnoses: definitions and classification 20032004. Philadelphia: NANDA; 2003.

(6) McCloskey JC, Bulechek G. Intervention Iowa Project. Nursing Interventions Classification. Saint Louis: Mosby Year-Book; 2000. 
(7) Johnson M, Maas M, Moorhead S. Nursing Outcomes Classification (NOC). 2nd ed. Saint Louis: Mosby Year-Book; 2000.

(8) Capra F. O ponto de mutação: a ciência, a sociedade e a cultura emergente. São Paulo: Cultrix; 1997.

(9) Dejours C. A loucura do trabalho: estudo da psicologia do trabalho. São Paulo: Cortez; 1988.

(10) Foucault M. Microfísica do poder. Rio de Janeiro: Graal; 1984.
(11) Goffman E. Manicômios, prisões e conventos. São Paulo: Perspectiva; 1974

(12) Vygostsky LS. A formação social da mente: o desenvolvimento dos processos psicológicos superiores. São Paulo: Martins Fontes; 1991.

(13) Carreteiro M. Construtivismo e educação. Porto Alegre: Artes Médicas; 1997.

(14) Perrenoud P. Novas competências para ensinar. Porto Alegre: Artmed; 2000
Programa de PósGraduação em Enfermagem na Sáude do Adulto da Escola de Enfermagem da USP

ANEXO

Quadro 1 - Articulação entre eixos temáticos e conjuntos de disciplinas

\begin{tabular}{|c|c|c|c|}
\hline \multirow[b]{2}{*}{$\begin{array}{c}\text { ELXOS } \\
\text { TEMÁTICOS }\end{array}$} & \multicolumn{3}{|c|}{ CONJUNTO DE DISCIPLINAS } \\
\hline & $\begin{array}{c}\text { Processos de } \\
\text { produção } \\
\text { de conhecimento }\end{array}$ & $\begin{array}{l}\text { Modelos teóricos sobre } \\
\text { o cuidar em } \\
\text { condições clínicas específicas }\end{array}$ & $\begin{array}{c}\text { Ensino } \\
\text { superior em } \\
\text { Enfermagem }\end{array}$ \\
\hline Cuidar clínico & $\begin{array}{l}\text { A história dos valores e } \\
\text { do pensamento } \\
\text { A investigação aplicada } \\
\text { à saúde do adulto } \\
\text { Método quantitativo de } \\
\text { pesquisa em } \\
\text { enfermagem } \\
\begin{array}{l}\text { Construção e adaptação } \\
\text { de instrumentos de } \\
\text { medida de variáveis } \\
\text { psicossociais aplicadas } \\
\text { na pesquisa em } \\
\text { enfermagem }\end{array} \\
\text { Bioética e o cuidar na } \\
\text { saúde do adulto }\end{array}$ & $\begin{array}{l}\text { Dimensão do cuidar do adulto no } \\
\text { contexto das doenças crônicas. } \\
\text { A psicossomática como perspectiva } \\
\text { para uma nova prática do } \\
\text { enfermeiro. } \\
\text { A reabilitação na assistência à } \\
\text { saúde do adulto. } \\
\text { Assistência ao ostomizado: da } \\
\text { teoria à prática. } \\
\text { A estomaterapia na prevenção e } \\
\text { tratamento de feridas. } \\
\text { Fundamentos teóricas de dor e de } \\
\text { sua avaliação. } \\
\text { Bases conceituais das } \\
\text { intervenções analgésicas. } \\
\text { Enfermagem na assistência à } \\
\text { pessoa com hipertensão arterial. } \\
\text { Fundamentos teóricos da } \\
\text { assistência à família e ao cuidador. } \\
\text { Cuidar em UTI: análise de suas } \\
\text { dimensões. } \\
\text { Processo de cuidar na } \\
\text { enfermagem perioperatória. } \\
\text { Enfermagem em neurociência. } \\
\text { Tópicos avançados de } \\
\text { enfermagem em neurotrauma. } \\
\text { A esfera do planejamento na } \\
\text { educação em saúde. }\end{array}$ & \\
\hline
\end{tabular}


Cibele A. de Mattos Pimenta Diná de A.L.M. da Cruz Kátia Grillo Padilha
Correspondência para: Cibele A. de M. Pimenta Av. Dr. Enéas de Carvalho Aguiar, 419 - Cerqueira César - São Paulo CEP - 05403-000 - SP (continuação)

\begin{tabular}{|c|c|c|c|}
\hline \multirow[b]{2}{*}{$\begin{array}{c}\text { EDXOS } \\
\text { TEMÁTICOS }\end{array}$} & \multicolumn{3}{|c|}{ CONJUNTO DE DISCIPLINAS } \\
\hline & $\begin{array}{c}\text { Processos de } \\
\text { produção } \\
\text { de conhecimento }\end{array}$ & $\begin{array}{c}\text { Modelos teóricos sobre } \\
\text { o cuidar em } \\
\text { condições clínicas específicas }\end{array}$ & $\begin{array}{c}\text { Ensino } \\
\text { superior em } \\
\text { Enfermagem }\end{array}$ \\
\hline & & $\begin{array}{l}\text { Bases teóricas em procedimentos } \\
\text { antimicrobianos. } \\
\text { Fundamentos de enfermagem I. } \\
\text { Fundamentos de enfermagem II. } \\
\text { Diagnóstico de enfermagem no } \\
\text { ensino, pesquisa e assistência ao } \\
\text { adulto. } \\
\text { Qualidade de vida na saúde do } \\
\text { adulto. } \\
\text { Tendências do cuidar no período } \\
\text { pós-operatório. }\end{array}$ & \\
\hline $\begin{array}{l}\text { Contexto do } \\
\text { cuidar }\end{array}$ & $\begin{array}{l}\text { A história dos valores e } \\
\text { do pensamento } \\
\text { A investigação aplicada } \\
\text { à saúde do adulto } \\
\text { Método quantitativo de } \\
\text { pesquisa em } \\
\text { enfermagem } \\
\text { Construção e adaptação } \\
\text { de instrumentos de } \\
\text { medida de variáveis } \\
\text { psicossociais aplicadas } \\
\text { na pesquisa em } \\
\text { enfermagem } \\
\text { Bioética e o cuidar na } \\
\text { saúde do adulto }\end{array}$ & $\begin{array}{l}\text { O cuidar e a dinâmica de } \\
\text { institucionalização da assistência } \\
\text { ao adulto } \\
\text { Stress, coping e trabalho. } \\
\text { Comunicação na saúde do adulto } \\
\text { I: a interação da linguagem verbal } \\
\text { e do não-verbal nas relações } \\
\text { interpessoais. } \\
\text { Comunicação na saúde do adulto } \\
\text { II: o não-verbal nas relações } \\
\text { interpessoais. } \\
\text { Tendências da prática do controle } \\
\text { de infecção hospitalar na } \\
\text { assistência de enfermagem. } \\
\text { Organização do trabalho em } \\
\text { saúde: objeto, tecnologias e } \\
\text { finalidades. }\end{array}$ & \\
\hline $\begin{array}{l}\text { Processos de } \\
\text { produção de } \\
\text { ensino } \\
\text { superior }\end{array}$ & $\begin{array}{l}\text { A história dos valores e } \\
\text { do pensamento } \\
\text { A investigação aplicada } \\
\text { à saúde do adulto } \\
\text { Método quantitativo de } \\
\text { pesquisa em } \\
\text { enfermagem } \\
\text { Construção e adaptação } \\
\text { de instrumentos de } \\
\text { medida de variáveis } \\
\text { psicossociais aplicadas } \\
\text { na pesquisa em } \\
\text { enfermagem } \\
\text { Bioética e o cuidar na } \\
\text { saúde do adulto }\end{array}$ & & $\begin{array}{l}\text { Educação em } \\
\text { enfermagem: } \\
\text { os princípios } \\
\text { constitutivos } \\
\text { da sua } \\
\text { identidade. }\end{array}$ \\
\hline
\end{tabular}

